\title{
A Study Based on Role of Amino Acids as Remedy for Nosocomial Bacterial Infections
}

\author{
Sandhya Khunger and Arpita Suri \\ Department of Biochemistry Shree Guru Gobind Singh \\ Tricentary University, Gurugram, Haryana, India \\ Corresponding author email: sandhya2_fmhs@sgtuniversity.org
}

\begin{abstract}
Antimicrobial resistance (AMR) is growing at an alarming rate leading towards becoming a threat to global health and development. According to WHO, AMR is one of the top ten global threats. Approximately 3 million antibiotic resistant infections occur in US annually with more than 35,000 deaths each year. The situation has attracted the attention of researchers towards searching for novel antibiotics and antimicrobial compounds. The present study is based on testing amino acids and their structures as novel therapy to target bacterial infections. it has been found that hydrophobic amino acids can be potential antibacterial compounds with mechanism of action based on bacterial cell membrane. The study provides low cost antibacterial compound that can be used as coating material or antimicrobial therapy for hospital setting to prevent AMR nosocomial infections in future.
\end{abstract}

KEY WORDS: ANTIBACTERIAL COMPOUND, ANTIMICROBIAL RESISTANCE, HYDROPHOBIC AMINO ACIDS, SURFACTANT.

\section{INTRODUCTION}

Amino acids and proteins are building units of human body. Proteins are essential for metabolic requirements of the body. Starting from superficial skin, a single strand of hair to metabolic enzymes, everything is made of proteins. Proteins are sequential collection of amino acids held together by peptide bonds. The amino acids are organic compounds made of two essential functional groups - amine and carboxylic groups along with a functional side chain. The presence of different side chain provides different properties to an individual amino acid. Humans, animals as well as microbes utilize these amino acids for their growth and development (Figure 1). Microbes including bacteria, viruses and fungi depend on amino acids and proteins for their existence. Moreover, microbes specifically bacteria have started using amino acids as defense mechanisms

Biosc Biotech Res Comm P-ISSN: 0974-6455 E-ISSN: 2321-4007

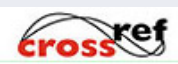

Identifiers and Pagination

Year: 2021 Vol: 14 No (6) Special Issue

Pages: $405-408$

This is an open access article under Creative

Commons License Attribn 4.0 Intl (CC-BY).

DOI: $h t t p: / / d x . d o i . o r g / 10.21786 / b b r c / 14.7 .85$ against adverse conditions such as physical shock, chemical treatment such as antibiotics (T. Defoirdt et. al. (2019)). The process requires maintenance of quorum sensing to accumulate other bacterial cells as a part of collective defense mechanism. The development of defense systems in bacteria has given rise to bacterial infections untreatable by common antibiotics. The ability of escaping antibacterial action of drugs by one or the other defense system is termed as multidrug resistance (MDR) or antimicrobial resistance (AMR).

AMR is most common in bacterial communities due to rapid evolution of metabolic systems in response to environmental conditions. The ubiquitous presence of bacteria proves the organism's ability to adapt extremely diverse environmental conditions including hot springs (thermophiles), chemically rich conditions; high salt conditions (halophiles), acidic conditions (acidophiles) etc. Interestingly, bacteria can adapt in complex environments such as effluent waste. There have been numerous studies where novel bacterial species have been isolated from waste effluent streams and cultured to develop industrially relevant biological organism capable of degrading complex chemicals (C. S. Karigar et. al. (2011)). Apart from this, bacteria species have been negatively associated with human infections. The organisms can cause severe diseases in humans such as cholera, leprosy,

\section{Article Information}

Received: $11^{\text {th }}$ May 2021

ccepted after revision: $26^{\text {th }}$ July 2021 
tuberculosis, plague,syphilis, anthrax etc. bacteria have been associated with severe skin and sexually transmitted diseases as well (N. T. Prabhat Shukla et. al. (2015)). The bacterial infections have acquired resistance to common antibiotics with time. The versatility of bacteria growth makes them survive antibacterial situations, which further grow into a pool of resistant bacteria leading to uncurable diseases (Figure 1).

The most alarming situation of bacterial infections is arising in nosocomial infections. Nosocomial infections are hospital acquired infections which develop within 72 hours of patient's admission. These create complications in health state of the patients more commonly in ICUs (intensive care units) (A. Asensio et. al. (2000)). Most of hospital acquired infections arise from endogenous bacterial cells; however, many critically sick patients become colonized with resistant bacteria. The infections have become leading cause of mortality in many ICUs, around $20-25 \%$ of nosocomial infections are caused by gram negative bacteria(R. G. Pearl et. al. (1992)). These bacterial cells are exposed to patients through mechanical ventilation including tracheal intubation causing aspiration of pathogens. The exponentially growing population of resistant bacteria has developed a threat in efficient treatment most of the severe infections occurring in humans. Thus, the present study provides a unique antibacterial source based on amino acids, which can be used as therapeutic strategy to treat bacterial infections in future.

Literature Review: The growing problem of antimicrobial resistance has developed a need to search for new antimicrobial compounds for targeting the resistance in bacterial infections. Cationic antimicrobial peptides (AMPs) have been used as promising alternatives in treating drug resistant bacterial infection. The major target of AMP includes bacterial cell membrane and some AMP penetrates cell membranes to target specific molecular pathway or cellular components. AMPs usually contain arginine and lysine residues to impart overall positive charge to the peptide(J. Wiesner et. al. (2010)). According to a study, amino acids based surfactants have been used in antimicrobial agents, drug delivery agents and viral vectors for gene therapy(M. R. Infante et. al. (2004)). The zwitter ionic property of the amino acids provides surfactant properties and helps in providing physical pressure over bacterial cells. Chican et al. conducted a study involving amino acid based surfactants (sodium lauroyl glycinate and sodium lauroyl sarcosinate) and amphoteric or nonionic surfactant to evaluate synergistic potential of aqueous surfactant system in terms of surface tension and foaming power. Lauryl dimethyl amine oxide was found to be synergistically acting with sodium lauroyl glycinate and sodium lauroyl sarcosinate producing better foaming and sulphate free formulation (E Chican et. al. (2019).

Surfactants display group of organic compounds having surface tension modification properties which can help in killing bacterial cells. Another study reported antibacterial properties through surfactants such as CTAB, CPC and BAC. The tested agents were found to be effective against gram positive as well as gram negative bacteria. Some surfactants showed selective effect towards P. vulgaris moreover, BAC was found better than other surfactants and effect was correlated with chemical structure of surfactants as well as properties of bacterial cell wall(K. K. Daria et. al. (2014)). The antibacterial therapies discussed so far represent peptide based surfactant based agents, which are complex form of pharmaceutical ingredients. The production process of these compounds requires time as well as complex technical instruments. Moreover, there is risk of toxicity when these compounds are used in human body. Thus there is need to develop simpler ingredients which minimal probability of human toxicity. The present study evaluates antibacterial properties of natural amino acids in order to provide a cost effective pharmaceutical ingredient for treating bacterial infections.

Table 1. Amino acids evaluated for antimicrobial activity in the present study.

\begin{tabular}{|l|c|c|}
\hline Abbreviation & Full name & Side chain features \\
\hline F & Phenylalanine & Hydrophobic benzyl side chain \\
\hline Y & Tyrosine & Benzyl side chain with hydroxyl reactive group, hydrophilic than F \\
\hline P & Proline & Side chain engaged in amino acid backbone \\
\hline S & Serine & Neutral non polar side chain \\
\hline L & Leucine & Hydrophobic aliphatic side chain \\
\hline N & Asparagine & Amide side chain \\
\hline
\end{tabular}

\section{Research Questions}

1. Can amino acids be used as novel antibacterial compounds to treat bacterial infections in humans?

2. What could be the probable mechanism of action behind the antibacterial property?

\section{MATERIAL AND METHODS}

Sample Collection: Bacterial species E. coli (gram negative) were procured from Institute of Microbial Technology (IMTECH, Chandigarh). All the amino acids (L-Phe, L-Tyr, L-Pro, L-Ser, L-Phe, L-Asn) used in the present study were purchased from Sisco Research Laboratories (Table 1). Luria Bertani (LB) broth and Soybean casein digest medium was purchase from Himedia. Triton-X was 
purchased from SDFCL (India). Statistical significance was evaluated through OriginPro Software and multiple sample comparison tests was performed by using one way ANOVA at threshold $\mathrm{p}<0.05$.

Preparation of amino acid solutions: Amino acids were prepared in $50 \mathrm{mM}$ sodium phosphate buffer $\mathrm{pH} 7$. The mixture was heated at $80 \mathrm{o} \mathrm{C}$ and further sonicated to obtain a clear solution. The solution was mixed vigorously over a vortex to break coagulated particles of amino acids powder. The final concentration of amino acids required is optimized accordingly in culture media. The sodium phosphate buffer without amino acids was used as control in further experiments.

Estimation of antibacterial activity: Cryo-cultures of E. coli bacteria were revived by primary culture of the strain in Luria Bertani (LB) media at $37 \mathrm{o} \mathrm{C,} 150 \mathrm{rpm}$. The primary culture at OD 0.6 to 0.8 is further diluted to obtain a secondary culture and incubated to obtain CFU $1 x 108$. To study antibacterial effect of amino acids, amino acids solution was added in media before inoculation and then CFU was calculated after 24 hours of incubation time exactly same as control (phosphate buffer).

Estimation of surface tension: The surface tension of amino acid solutions was determined at room temperature through contact angle goniometer pendant drop method. Briefly, water, buffer and amino acid solutions (AA) were taken in syringe and pendant like drop was formed which was captured by charged coupled device for capturing image. The image was used as reference to evaluate surface tension.

Membrane destabilization assay: Amino acids were subjected to membrane destabilization assay to determine their capability in disrupting cell membrane. The assay was done by preparing model membrane of lecithin. The membrane was formulated as vesicles containing rhodamine dye. Briefly, the vesicles were prepared by preparing a solution of lecithin $(15 \mathrm{mg} / \mathrm{ml})$ in chloroform and methanol $(9: 1)$. The solution was vaccum evaporated to prepare a thin film. The rhodamine dye solution $(5 \mathrm{mg} / \mathrm{ml})$ was added to the film and solution was sonicated for 1.5 hours. The size of vesicles formed was determined by Dynamic Light Scattering. The amino acid solution was added in the vesicles and rhodamine dye release was measured by fluorimetry, determining absorbance at 560nm.

Hemolysis assay: Blood was collected from three volunteers and stored in AcCuvet-Plus K3-EDTA tube and centrifuged at $1000 \mathrm{~g}$ for 10 minutes at $4 \mathrm{o} \mathrm{C}$. The erythrocytes accumulated at tube's bottom were provided with different amino acids solutions. The suspension was centrifuged at $10,000 \mathrm{~g}$ for $10 \mathrm{~min}$ and absorbance of supernatant was recorded at $540 \mathrm{~nm}$. The percent hemolysis was calculated by formula mentioned below.

$$
\text { Hemolysis }(\%)=\frac{A_{540}(\text { sample })-A_{540} \text { (negative control) }}{A_{540}\left(\text { positive control) }-A_{540}\right. \text { (negative control) }}
$$

\section{RESULTS AND DISCUSSION}

Antibacterial assay: Antibacterial activity of amino acids was estimated as mentioned previously over Gram negative bacteria E. coli. L-Ser, L-Phe and L-Asn were able to reduce cell survival from $95 \%$ to approximately $50 \%$. L-Ser consists of hydromethyl group which has been reported in exhibiting surfactant properties as well. L-Phe consists of aromatic side chain with moderate flexibility which may lead to hydrophobic interactions leading to physical pressure over cell membrane. L-Asn displays least antibacterial activity which can be attributed to its ability to reduce surface tension. Amino acids have been reported to lower surface tension in solution form which may be the reason for antibacterial activity in both ram positive as well as gram negative bacteria.

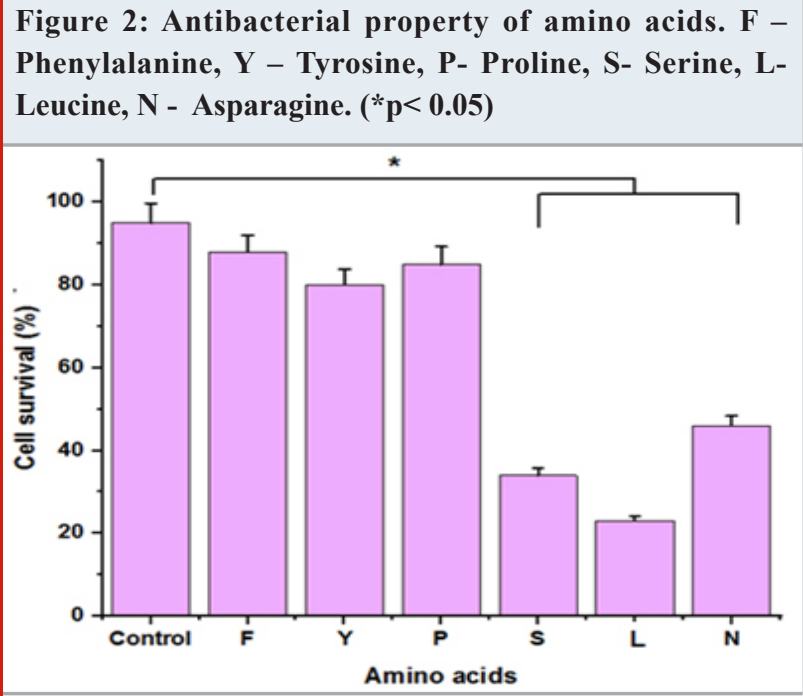

Surface tension analysis: Surface tension hold importance in case of surfactant compounds which reduced interfacial distance between water molecules. Surfactant molecules display intermolecular bonding to form micelles and provide shear force as well as interaction based attraction of elements inside the molecular core. Some of amino acids have been known for having surfactant like properties which can provide essential shear force to kill bacterial cells. Thus, surface tension of amino acids was estimated to correlate the mechanism of cell killing based on cell membrane. Referring to Figure 2 and Figure 3, it can be marked out that amino acids Ser (S), Leu (L) and Asn (N) show antibacterial properties which can be attributed to their property of lowering the surface tension. These amino acids may exert shear force on the bacterial cell membrane and cause cell killing. The surfactant property of these amino acids can be further utilized in cleaning of biomedical devices including catheters, implants, and surgical instruments etc. for preventing the risk of bacterial infections to the patients. Moreover the property can be used to create formulations and coatings for prevention of biofilm formation in future.

Membrane destabilization assay: In order to find the mechanism of antibacterial action, membrane destabilization was performed to test if the action mechanism is based on 
cell membrane lysis. Membrane models were made and filled with rhodamine dye. Membrane destabilization was studied when dye is secreted in the solution, leading to rise in absorbance at dye maxima. As seen in Figure 4, absorbance was observed in L-Ser, L-Leu and L- Asn. However, L-Phe, L-Tyr and L-Pro showed insignificant difference in dye release as compared to control. Thus it can be concluded that $\mathrm{S}, \mathrm{L}$ and $\mathrm{N}$ are causing rupture in membrane model leading to dye release in the solution. Overall, the results suggest that mechanism of action behind antimicrobial activity of S, L and $\mathrm{N}$ may be based on targeting bacterial membrane and causing cell killing. Further studies could be done where ruptured membrane can be observed in Scanning electron microscope, to confirm the results in future.

Figure 3: Reduction of surface tension displayed by amino acids. F - Phenylalanine, Y - Tyrosine, P- Proline, S- Serine, L- Leucine, $\mathrm{N}$ - Asparagine. $\left({ }^{*} \mathbf{p}<0.05\right)$

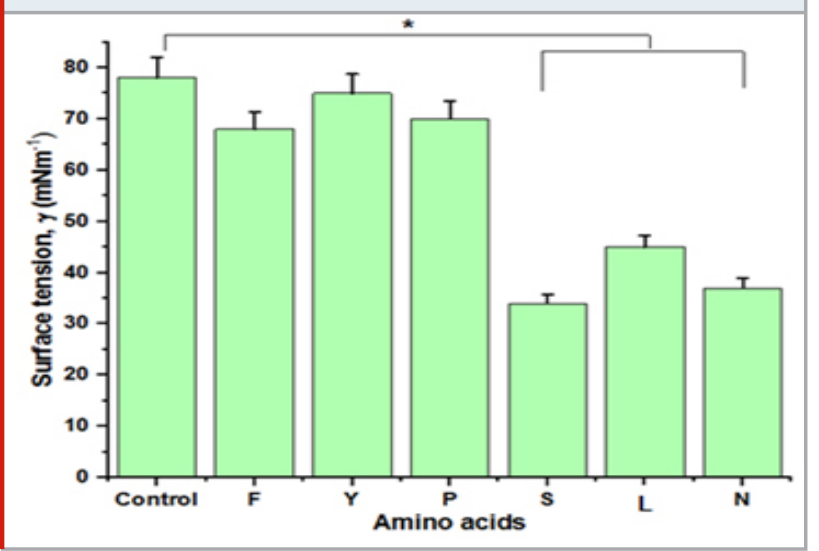

Figure 4: a) Membrane destabilization shown by different amino acids. b) Disruption of human red blood cells shown by different amino acids. F - Phenylalanine, Y - Tyrosine, P- Proline, S- Serine, L- Leucine, N - Asparagine.
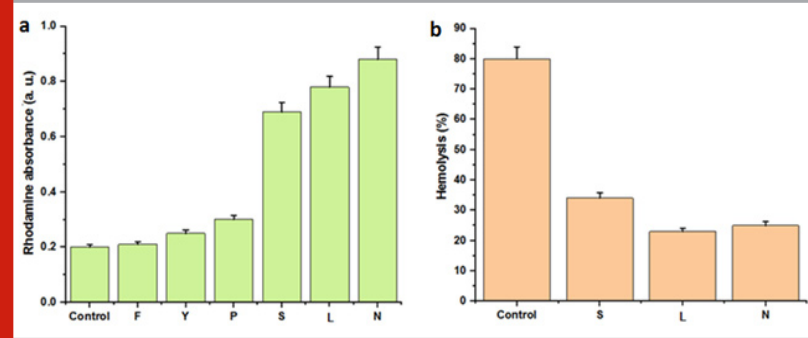

Hemolysis assay: As per the results discussed till now, it has been found that amino acids $\mathrm{S}, \mathrm{L}$ and $\mathrm{N}$ exhibit antibacterial activity with probable mechanism of action based on bacterial cell membrane. Further, it is necessary to evaluate cytotoxicity of these amino acids to enhance their applicability. Hemolysis assay was performed where PBS was used as negative control and triton $-\mathrm{X}$ was used as positive control. The study was conducted in human red blood cells and it was found that all three amino acids $\mathrm{S}, \mathrm{L}$ and $\mathrm{N}$ show less lysis of RBCs. S exhibits maximum hemolysis among other amino acids but still less than triton-X. This shows that these amino acids can possess compatibility with hum blood cells, making their application reliable. Further, in-vivo studies can be performed to confirm the non-cytotoxicity of these amino acids in future experiments.

\section{CONCLUSION}

The most common form of infections found in humans is bacterial infections which affect the body in terms of underlying diseases. The researchers have been trying to develop novel strategies to treat bacterial infection. The versatility and diversification of bacterial species has made them resistant towards most of the available antibiotic therapies. Thus there is need to develop strategies to prevent and treat diseases caused by bacterial species. The ability of humans to use these amino acids naturally for defense against diseases and even bacterial species used amino acids for saving them from external stress caused by other organisms. Moreover studies have suggested that bacterial species release amino acids to detach themselves from aggregated community of microbes to travel from one location to other.

Thus, it was hypothesized that these amino acids can display antibacterial properties. The present study has evaluated amino acids as potent antibacterial compounds. It has been found that mostly hydrophobic amino acids are capable of producing antibacterial action. The property can be attributed to their surfactant action and their capability to breakdown model membrane. Thus, these amino acids can exert their action on bacterial cell membrane leading to cell killing. Overall, the study provides a scope of finding new antimicrobial compounds for fighting the crises of antibiotics created by multi drug resistant bacteria.

\section{REFERENCES}

Asensio, A., Oliver, A., González-Diego, P., Baquero, F., Pérez-Díaz, J.C., Ros, P., Cobo, J., Palacios, M., Lasheras, D. and Cantón, R., 2000. Outbreak of a multiresistant Klebsiella pneumoniae strain in an intensive care unit: antibiotic use as risk factor for colonization and infection. Clinical Infectious Diseases, 30(1), pp.55-60.

Butterworth, J.F., Mackey, D.C. and Wasnick, J.D., 2013. Morgan \& Mikhail's clinical anesthesiology (Vol. 15). New York: McGraw-Hill.

Defoirdt, T., 2019. Amino acid-derived quorum sensing molecules controlling the virulence of vibrios (and beyond). PLoS pathogens, 15(7), p.e1007815.

Infante, M.R. and Perez, L., 2004. PINAZO AURORA, et al. Amino acid-based suffaetants, 7(6-7), pp.583-592.

Karigar, C.S. and Rao, S.S., 2011. Role of microbial enzymes in the bioremediation of pollutants: a review. Enzyme Res 2011: 1.

Wiesner, J. and Vilcinskas, A., 2010. Antimicrobial peptides: the ancient arm of the human immune system. Virulence 1: 440-464.

Wieczorek, D., Gwiazdowska, D., Michocka, K., Kwaśniewska, D. and Kluczyńska, K., 2014. Antibacterial activity of selected surfactants. Polish J Commodity Sci, 2(38), pp.142-9. 